SLAC-PUB-13219

arXiv:0804.2529v1[astro-ph]

April 2008

\title{
Suzaku observation of TeV blazar the 1ES 1218+304: clues on particle acceleration in an extreme $\mathrm{TeV}$ blazar
}

\author{
R. Sato ${ }^{1}$, J. Kataoka ${ }^{2}$, T. Takahashi ${ }^{1}$, G. M. Madejski ${ }^{3}$, S. Rügamer ${ }^{4}$ and S. J. Wagner ${ }^{5}$
}

\begin{abstract}
We observed the TeV blazar 1ES 1218+304 with the X-ray astronomy satellite Suzaku in May 2006. At the beginning of the two-day continuous observation, we detected a large flare in which the $5-10 \mathrm{keV}$ flux changed by a factor of $\sim 2$ on a timescale of $5 \times 10^{4} \mathrm{~s}$. During the flare, the increase in the hard X-ray flux clearly lagged behind that observed in the soft X-rays, with the maximum lag of $2.3 \times 10^{4}$ s observed between the $0.3-1 \mathrm{keV}$ and $5-10 \mathrm{keV}$ bands. Furthermore we discovered that the temporal profile of the flare clearly changes with energy, being more symmetric at higher energies. From the spectral fitting of multiwavelength data assuming a one-zone, homogeneous synchrotron self-Compton model, we obtain $B \sim 0.047 \mathrm{G}$, emission region size $R=3.0 \times 10^{16} \mathrm{~cm}$ for an appropriate beaming with a Doppler factor of $\delta=20$. This value of $B$ is in good agreement with an independent estimate through the model fit to the observed time lag ascribing the energy-dependent variability to differential acceleration timescale of relativistic electrons provided that the gyro-factor $\xi$ is $10^{5}$.
\end{abstract}

Subject headings: BL Lacerate objects: individual (1ES 1218+304) - radiation mechanisms: non-thermal - X-rays: galaxies

\footnotetext{
${ }^{1}$ Institute of Space and Astronautical Science/JAXA, Sagamihara, Kanagawa 229-8510, Japan

${ }^{2}$ Department of Physics, Tokyo Institute of Technology, Meguro-ku, Tokyo 152-8551, Japan

${ }^{3}$ Stanford Linear Accelerator Center and Kavli Institute for Particle Astrophysics and Cosmology, Stanford University, Stanford, CA 94305, USA

${ }^{4}$ Universität Würzburg, Am Hubland, D-97074 Würzburg, Germany

${ }^{5}$ Landessternwarte, Universität Heidelberg, Königstuhl, 69117 Heidelberg, Germany
}

Submitted to Astrophysical Journal Letters

Work supported in part by US Department of Energy contract DE-AC02-76SF00515 


\section{Introduction}

Blazars are a sub-category of Active Galactic Nuclei where a relativistic jet pointing close to our line of sight produces Doppler-boosted emission (e.g., Urry \& Padovani 1995; Ulrich, Maraschi \& Urry 1997). Generally, their overall spectra have two pronounced continuum components: one peaking between IR and X-rays is produced by the synchrotron radiation of relativistic electrons, and the other in the $\gamma$-ray regime, presumably due to the inverse Compton (IC) emission by the same electrons. In some cases, $\gamma$-ray emission is seen to extend to the $\mathrm{TeV}$ range; the $\mathrm{X}$-ray and $\mathrm{GeV} / \mathrm{TeV} \gamma$-ray bands correspond to the highest energy ends $\left(E_{\max }\right)$ of the synchrotron/IC emission (e.g., Inoue \& Takahara 1996; Kirk, Rieger \& Mastichiadis 1998). At these ends, variability is expected to be most pronounced, and in fact, such large flux variations are observed, on a timescale of hours to days (e.g., Kataoka et al. 2001; Tanihata et al. 2001) or even shorter (minutes scale; Aharonian et al. 2007; Albert et al. 2007). Using ASCA data, Takahashi et al. (1996) argued the soft X-ray $(<1 \mathrm{keV})$ variation of Mrk 421, observed to lag behind that of the hard X-rays $(\geq 2$ $\mathrm{keV}$ ) by $\sim 4 \mathrm{ks}$, may well be ascribed to the energy dependence of the synchrotron cooling timescale. More recently, Kataoka et al. (2000) interpreted an observed soft-lag and spectral evolution of PKS 2155-304 by a newly developed time-dependent synchrotron self-Compton (SSC) model.

The above paradigm of "soft-lag" was questioned, however, in several aspects. First, intensive X-ray monitoring of blazars has revealed not only soft lags but in some cases hard lags (Takahashi et al. 2000) which may be a manifestation of another process, e.g., energy dependent acceleration. Second, Edelson et al. (2001) voiced concerns about reliability of measurement of lags that are smaller than the orbital periods $(\sim 6 \mathrm{ks})$ of low Earth orbit satellites. This was refuted by Tanihata et al. (2001) and Zhang et al. (2004) who showed that, although periodic gaps introduce larger uncertainties than evenly sampled data, lags on hour-scale cannot be the result of periodic gaps. A time resolved cross correlation analysis of uninterrupted Mrk 421 data obtained by XMM-Newton revealed lags of both signs, changing on time scales of up to a few $10^{3} \mathrm{~s}$ (Brinkmann et al. 2005). Hence the situation is very complex and still under debate.

In this letter we present new results from the May 2006 Suzaku observation of 1ES 1218+304 conducted as part of a multi-wavelength campaign with KVA-Swift-MAGIC. 1ES 1218+304 is categorized as a high-frequency peaked BL Lac object, at a redshift $z=0.182$ (VeronCetty \& Veron 2003). It was discovered as a TeV emitter by MAGIC at energies $>100 \mathrm{GeV}$ (Albert et al. 2006) and subsequently confirmed by VERITAS (Fortin 2007). While the detailed multiband analysis is ongoing, we focus in this letter on a remarkable X-ray flare observed with Suzaku. We present temporal and spectral features in $\S 3$, in $\S 4$ we discuss 
a physical origin of temporal variability.

\section{Observation and Data Reduction}

1ES 1218+304 was observed with Suzaku (Mitsuda et al. 2007) during 2006 May 20-21 UT, yielding a net exposure time of $79.9 \mathrm{ks}$. Suzaku carries four sets of X-ray telescopes (Serlemitsos et al. 2007) each with a focal-plane X-ray CCD camera (XIS, X-ray Imaging Spectrometer; Koyama et al. 2007) that is sensitive over the 0.3-12 keV band, together with a non-imaging Hard X-ray Detector (HXD; Takahashi et al. 2007; Kokubun et al. 2007), which covers the 10-600 keV energy band with Si PIN photo-diodes and GSO scintillation detectors. 1ES 1218+304 was focused on the nominal center position of the HXD detector.

For the XIS, we analyzed the screened data, reduced via Suzaku software version 2.0. The screening was based on the following criteria: (1) only ASCA-grade 0,2,3,4,6 events were accumulated, while hot and flickering pixels were removed using the CLEANSIS script, (2) the time interval after the passage of South Atlantic Anomaly is greater than $500 \mathrm{~s}$, (3) the object is at least $5^{\circ}$ and $20^{\circ}$ above the rim of the Earth (ELV) during night and day, respectively. In addition, we also select the data with a cutoff rigidity (COR) larger than $6 \mathrm{GV}$. After this screening, the net exposure for good time intervals is $69.4 \mathrm{ks}$. The XIS events were extracted from a circular region with a radius of $4.2^{\prime}$ centered on the source peak, whereas the background was accumulated in an annulus with inner and outer radii of $5.4^{\prime}$ and $7.3^{\prime}$, respectively. We checked that the use of different source and background regions did not affect the analysis results. The response and auxiliary files are produced using the analysis tools XISRMFGEN and XISSIMARFGEN developed by the Suzaku team, which are included in the software package HEAsoft version 6.4.

The HXD/PIN data (version 2.0) were processed with basically the same screening criteria as those for the XIS, except that ELV $\geq 5^{\circ}$ through night and day and COR $\geq 8 \mathrm{GV}$. The HXD/PIN instrumental background spectra were provided by the HXD team for each observation (Kokubun et al. 2007; Fukazawa et al. 2006). Both the source and background spectra were made with identical good time intervals and the exposure was corrected for detector deadtime of 6.0\%. We used the response files version AE_HXD_PINHXDNOM2_20070914.RSP, provided by the HXD team. 


\section{Analysis and Results}

Figure 1 shows the averaged light curves of the four XISs in the six X-ray energy bands. Although we could see variations of count rates at some level using HXD/PIN data, it was not significant within uncertainties of photon statistics. Thus in the following, we concentrate on the temporal variability of the XIS data only, below $10 \mathrm{keV}$. The temporal variation of the hardness ratio (HR) is also shown in the bottom panel of Figure 1. It indicates that the variability in the soft and hard X-ray bands are not well synchronized.

To quantify the different shape of the flare with energy dependent time-lags, we fitted the light curves with a function given by Norris (1996) after a slight modification of adding a constant offset $C_{0}$ to mimic the observed light curves:

$$
\begin{aligned}
I(t) & =C_{0}+C_{1} \times \exp \left[-\left(\left|t-t_{\text {peak }} / \sigma_{\mathrm{r}}\right|\right)^{k}\right] \quad\left(\text { for } t \leq t_{\text {peak }}\right), \\
& =C_{0}+C_{1} \times \exp \left[-\left(\left|t-t_{\text {peak }} / \sigma_{\mathrm{d}}\right|\right)^{k}\right] \quad\left(\text { for } t>t_{\text {peak }}\right),
\end{aligned}
$$

where $t_{\text {peak }}$ is the time of the flare's maximum intensity $C_{1}, k$ is a measure of pulse sharpness, $\sigma_{\mathrm{r}}$ and $\sigma_{\mathrm{d}}$ are the rise and decay time constants. If the light curve is symmetric in time, $\sigma_{\mathrm{r}}$ and $\sigma_{\mathrm{d}}$ are expected to be equal. All the light curves were binned at $2880 \mathrm{~s}$ (a half of the orbital period of Suzaku) for fitting. The results of the fittings are given in Table 1. In summary, the observed flare shows the following characteristics: (1) The flare shape is asymmetric in time $\left(\sigma_{\mathrm{r}} / \sigma_{\mathrm{d}}<1\right)$ especially in the lower energy band (but note $\sigma_{\mathrm{r}} / \sigma_{\mathrm{d}} \simeq 1$ for $5-10 \mathrm{keV}$ light curve). (2) The flare amplitude defined as $\left(C_{1}+C_{0}\right) / C_{0}$ becomes larger as the photon energy increases (the $5-10 \mathrm{keV}$ flux changed by a factor of $\sim 2$ ). (3) The rise-time of the flare is almost constant $\sim 5 \times 10^{4} \mathrm{~s}$ below $2 \mathrm{keV}$, while it becomes gradually longer at higher energy bands.

Next, we try to evaluate lags of temporal variations in various energy bands. Taking into account a wide variety of the flare shape measured at different energies, we estimated lags by just comparing the peak-time of the flare rather than using other temporal techniques, such as the discrete correlation function (DCF; Edelson \& Krolik 1989) or the modified mean deviation method (MMD; Hufnagel \& Bregman 1992). 1 We compared the peak-time in five lower energy bands to that determined in the $5-10 \mathrm{keV}$ band. Apparently, the hard X-ray $(5-10 \mathrm{keV})$ peak lagged behind that in the soft X-ray $(0.3-1 \mathrm{keV})$ by $(2.3 \pm 0.7) \times 10^{4}$ s. Importantly, this is much larger than the orbital period of Suzaku and less affected by artifacts proposed in Edelson et al. (2001).

\footnotetext{
${ }^{1}$ Since the DCF quantifies the degree of similarity or correlation between two time series as a function of the time-lag, it is not suitable to evaluate "energy-dependent" profiles, as observed in 1ES 1218+304.
} 


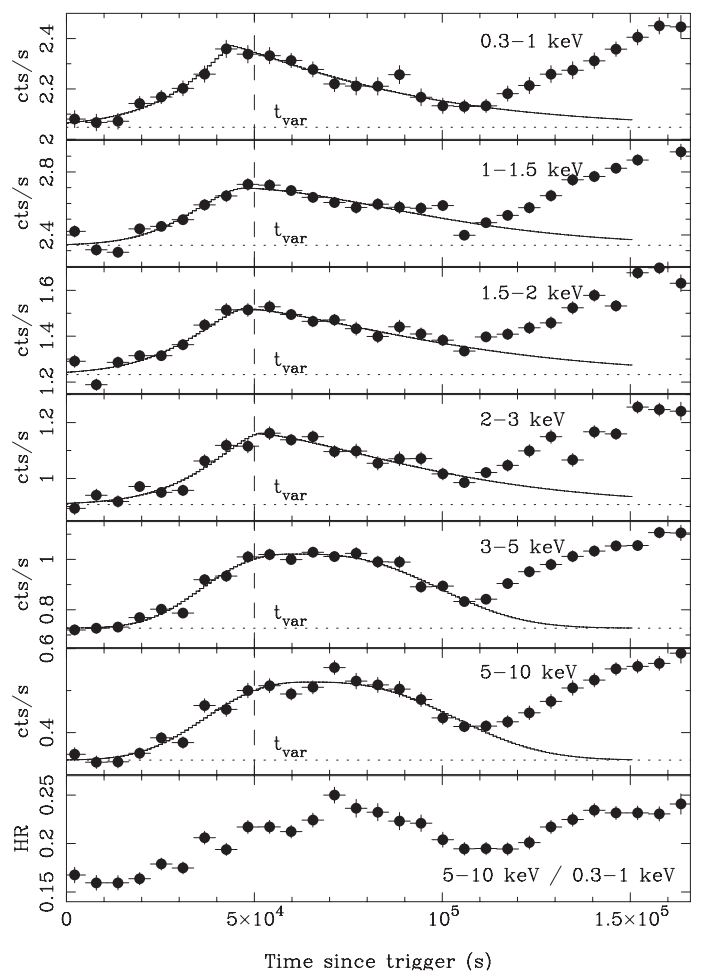

Fig. 1. - Light curves of 1ES 1218+304 observed with Suzaku XISs in 2006 May. The energy bands are $0.3-1,1-1.5,1.5-2,2-3,3-5$ and $5-10 \mathrm{keV}$ (from the upper panel), respectively. The bottom panel shows the $\mathrm{HR}$ of count rates, defined as $(5-10 \mathrm{keV}) /(0.3-1 \mathrm{keV})$. The dotted line is the constant offset $C_{0}$. The dashed line is the characteristic variability time scale of a flare $t_{\mathrm{var}} \simeq 5 \times 10^{4} \mathrm{~s}$.

The time averaged four XISs and HXD/PIN background subtracted spectra were fitted using XSPEC ver.11.3.2, including data within the energy band 0.6-50 keV. The background of HXD/PIN includes both the instrumental (non X-ray) background and the contribution from the cosmic X-ray background (CXB; Gruber et al. 1999). Here the form of the CXB was taken as $9.0 \times 10^{-9}(E / 3 \mathrm{keV})^{-0.29} \exp (-E / 40 \mathrm{keV}) \operatorname{erg~} \mathrm{cm}^{-2} \mathrm{~s}^{-1} \mathrm{sr}^{-1} \mathrm{keV}^{-1}$ and the observed spectrum was simulated assuming the PIN detector response to isotropic diffuse emission. We first fitted with a single power-law model with Galactic absorption $N_{\mathrm{H}}=1.78 \times 10^{20}$ $\mathrm{cm}^{-2}$ (Costamante et al. 2001). We obtained the best fit photon index $\Gamma=2.14 \pm 0.01$, but this model did not represent the spectrum well yielding a reduced $\chi^{2}$ of 1.23 for 1967 dof. We also tried to fit with a broken power-law model with Galactic absorption. The photon index below the break energy $E_{\text {brk }}\left(\Gamma_{1}\right)$ is $2.04 \pm 0.01$ while the index above $E_{\text {brk }}$ is $2.17 \pm 0.01$, where $E_{\text {brk }}\left(\Gamma_{2}\right)$ is $1.42 \pm 0.05 \mathrm{keV}$. The flux over $2-10 \mathrm{keV}$ is $\sim 2.0 \times 10^{-11} \mathrm{erg}$ $\mathrm{cm}^{-2} \mathrm{~s}^{-1}$. This model gives a better fit with a reduced $\chi^{2}$ of 1.14 for 1959 dof compared to the single power-law model, but $\chi^{2}$ is still not acceptable. Considering the spectral variability, 
Table 1: Temporal profiles during the flare of 1ES $1218+304$ in various X-ray energy bands.

\begin{tabular}{lcccc}
\hline $\mathrm{E}(\mathrm{keV})$ & $t_{\text {peak }}\left(10^{4} \mathrm{~s}\right)$ & $\sigma_{\mathrm{r}} / \sigma_{\mathrm{d}}$ & $k$ & $\left(C_{1}+C_{0}\right) / C_{0}$ \\
\hline $0.3-1$ & $4.4 \pm 0.1$ & $0.34 \pm 0.14$ & $1.2 \pm 0.4$ & $1.2 \pm 0.1$ \\
$1-1.5$ & $4.7 \pm 0.3$ & $0.31 \pm 0.08$ & $1.7 \pm 0.5$ & $1.2 \pm 0.1$ \\
$1.5-2$ & $4.7 \pm 0.3$ & $0.31 \pm 0.11$ & $1.4 \pm 0.5$ & $1.2 \pm 0.1$ \\
$2-3$ & $5.1 \pm 0.3$ & $0.33 \pm 0.10$ & $1.4 \pm 0.4$ & $1.3 \pm 0.1$ \\
$3-5$ & $6.1 \pm 0.4$ & $0.67 \pm 0.12$ & $2.7 \pm 0.4$ & $1.4 \pm 0.1$ \\
$5-10$ & $6.7 \pm 0.7$ & $0.84 \pm 0.17$ & $2.8 \pm 0.6$ & $1.6 \pm 0.1$ \\
\hline
\end{tabular}

we analyzed the spectrum every $5760 \mathrm{~s}$. The power-law indices vary from $2.05 \pm 0.01$ to $2.22 \pm 0.01$ during the flare, and each segment can be fitted well with a single power-law model or broken power-law model with $\chi^{2} /$ dof ranging from 0.94 to 1.09 .

Figure 2 shows the spectral energy distribution (SED) of 1ES 1218+304 with currently available datasets. The TeV data are obtained from Albert et al. (2006), and are corrected for the absorption due to the IR Extragalactic background light (EBL; see Fig.2); other data are from the NED database. Note that the $\mathrm{TeV}$ analysis of multi-wavelength campaign data is still ongoing and the combined datasets will be investigated in forthcoming paper (Stefan et al. in prep). As expected from the curved X-ray spectrum with photon index $\Gamma$ around 2 and $E_{\text {cut }} \lesssim 10 \mathrm{keV}$, the synchrotron emission peaks just around the Suzaku bandpass.

In order to specify the SED of 1ES 1218+304, we applied a one-zone homogeneous SSC model developed in Kataoka et al. (1999). Noting that the characteristic variability time scale of the flare is $t_{\mathrm{var}} \simeq 5 \times 10^{4} \mathrm{~s}$, which is most probably determined by the light travel time across the source emitting region (see discussion in $\S 4$ ), we obtain $R=c t_{\mathrm{var}} \delta=$ $3.0 \times 10^{16} \mathrm{~cm}$ for a moderate beaming factor of $\delta=20$ (e.g., Kataoka et al. 1999; 2000 for self-consistent determination of physical parameters in TeV blazars). With this parameter set, the SED of $1 \mathrm{ES} 1218+304$ is fitted with $B=0.047 \mathrm{G}, s=1.7, \gamma_{\min }=1, \gamma_{\mathrm{brk}}=8.0 \times 10^{3}$ and $\gamma_{\max }=8.0 \times 10^{5}$. We also note that the energy densities of electrons and fields are $u_{e}=8.3 \times 10^{-3} \mathrm{erg} / \mathrm{cm}^{3}$ and $u_{B}=8.8 \times 10^{-5} \mathrm{erg} / \mathrm{cm}^{3}$, respectively. Thus the jet in $1 \mathrm{ES} 1218+304$ is particle dominated, and the ratio $u_{e} / u_{B} \sim 100$ is well within the range of typical TeV blazars.

\section{Discussion}

In our observation we detected a large flare during which the hard X-ray variation lagged behind that in the soft X-rays, $\sim 2.3 \times 10^{4} \mathrm{~s}$. This is completely opposite to a well-known 


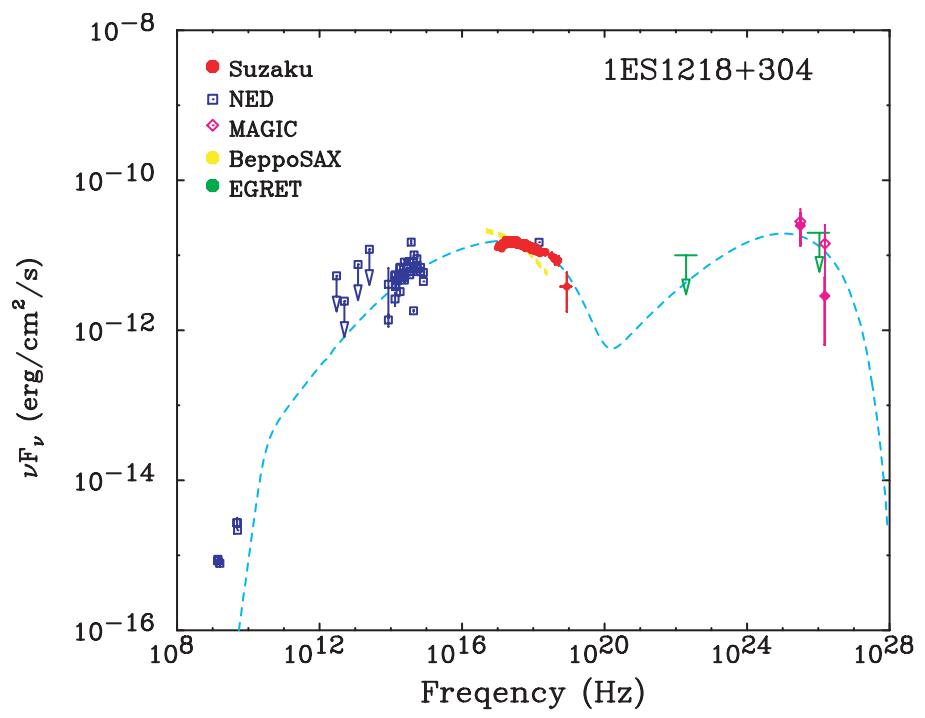

Fig. 2.- Overall SED of 1ES 1218+304. Filled circles show X-ray data (Suzaku; this work). For TeV data (Albert et al. 2006; filled diamonds), we adopt the correction for the IR EBL by Primack et al. 2001 (open diamonds); see also Primack et al. (2005) as well as the discussion based on the recent HESS detection of distant TeV blazars (Costamante 2007). The other plots are from the NED database. The dashed line is a prediction by a one-zone SSC model (Kataoka et al. 2000).

behavior in which the spectra harden during the phases of rising flux, and soften during the phases of falling flux, as has been obtained from the past observations. In the theoretical context, however, "hard lag" is actually expected especially in the X-ray variability of $\mathrm{TeV}$ blazars, but has never been observed so clearly before. It has been suggested that a hard-lag is observable only at energies closer to the maximum electron energy, $\gamma_{\max }$ (Kirk, Rieger \& Mastichiadis 1998), where the acceleration time is almost comparable to the cooling time scale of radiating electrons: $t_{\mathrm{acc}}\left(\gamma_{\max }\right) \simeq t_{\mathrm{cool}}\left(\gamma_{\max }\right)$.

It is convenient to express $t_{\text {acc }}$ and $t_{\text {cool }}$ in terms of the observed photon energy $E$ (in units of $\mathrm{keV}$ ). Noting that the typical synchrotron emission frequency, averaged over pitch angles, of an electron with energy $\gamma m c^{2}$ is given by $\nu \sim 3.7 \times 10^{6} \mathrm{~B} \gamma^{2} \mathrm{~Hz}$, we obtain;

$$
\begin{aligned}
t_{\mathrm{acc}}(E) & =9.65 \times 10^{-2}(1+z)^{3 / 2} \xi B^{-3 / 2} \delta^{-3 / 2} E^{1 / 2} \mathrm{~s}, \\
t_{\mathrm{cool}}(E) & =3.04 \times 10^{+3}(1+z)^{1 / 2} B^{-3 / 2} \delta^{-1 / 2} E^{-1 / 2} \mathrm{~s},
\end{aligned}
$$

where $z$ is the redshift, $B$ is the magnetic field strength, $\xi$ is the "gyro-factor" which can be identified with the ratio of energy in an ordered magnetic field to that in a turbulent magnetic field ( $\xi=1$ for the Bohm limit; see, e.g., Inoue \& Takahara 1996), and $\delta$ is the beaming factor. Note that for lower energy photons, $t_{\text {acc }}(E)$ is always shorter than $t_{\text {cool }}(E)$ 
because higher energy electrons need longer time to be accelerated $\left(t_{\text {acc }}(\gamma) \propto \gamma\right)$ but cool rapidly $\left(t_{\text {cool }}(\gamma) \propto \gamma^{-1}\right)$. This energy dependence of acceleration/cooling time-scales may qualitatively explain the observed characteristics of the X-ray light curves of 1ES 1218+304. It is thus interesting to consider a simple toy model in which the rise time of the flare is primarily controlled by the acceleration time of the electrons corresponding to observed photon energies, while the fall time of the flare is due to the synchrotron cooling time scale. In this model, the amount of "hard-lag", $\tau_{\text {hard }}$, is simply due to the difference of $t_{\text {acc }}$, and independent of the energy dependence of $t_{\text {cool }}$ :

$$
\begin{aligned}
\tau_{\text {hard }} & =t_{\mathrm{acc}}\left(E_{\mathrm{hi}}\right)-t_{\mathrm{acc}}\left(E_{\mathrm{low}}\right) \\
& \sim 9.65 \times 10^{-2}(1+z)^{3 / 2} \xi B^{-3 / 2} \delta^{-3 / 2}\left(E_{\mathrm{hi}}^{1 / 2}-E_{\mathrm{low}}^{1 / 2}\right) \mathrm{s},
\end{aligned}
$$

where $E_{\text {low }}$ and $E_{\text {hi }}$ are the lower and higher X-ray photon energies to which the time-lag is observed. Here we took $E_{\text {low/hi }}$ to be the logarithmic mean energy in the observation energy bandpass. The result of the model fit to the observed $\tau_{\text {hard }}$ is shown in Figure 3 (left).

Assuming a beaming factor $\delta=20$ from multiband spectral fitting (see $\S 3$ ), the best fit parameter of the magnetic field $B$ can be written as $\sim 0.049 \xi_{5} \mathrm{G}$, where $\xi_{5}$ is the "gyrofactor" in units of $10^{5}$. Thus, in order to have the $B$ field required in the acceleration region consistent with that derived from the SED fitting, we infer $\xi \sim 10^{5}$. Such high value of $\xi$ is in fact consistent with that inferred by Inoue \& Takahara (1996) for other blazars. With these parameters, the maximum synchrotron radiation energy $E_{\max }$, corresponding to $\gamma_{\max }$, is expected to be $\sim 5.3 \mathrm{keV}$. Hence, the above toy model qualitatively well represents the observed spectral/temporal features of 1ES 1218+304, in particular: (1) the synchrotron component peaks around the Suzaku XIS energy band in the multiband spectrum (Figure 2) and (2) the observed light curve is symmetric in shape when measured at the high energy band, while being "asymmetric" (i.e., fall time longer than the rise time) at the lower energy band. Figure 3 (right) compares the energy dependence of observed and modeled flare shapes, defined as the ratio of rise and decay time-scales, $\sigma_{\mathrm{r}} / \sigma_{\mathrm{d}}$. The dashed line shows the model prediction from $\sigma_{\mathrm{r}} / \sigma_{\mathrm{d}} \simeq t_{\text {acc }} / t_{\text {cool }}=\left(E / E_{\max }\right)^{1 / 2} /\left(E / E_{\max }\right)^{-1 / 2} \sim E / 5.3 \mathrm{keV}$. Although the general trend is well reproduced, Figure 3 indicates that the observed rise time may have a bit longer time scale than expected from the model. The most natural interpretation for this is the smoothing of rapid variability by the source light crossing time scale $t_{\text {crs }}$ (e.g., Chiaberge \& Ghisellini 1999; Kataoka et al. 2000). Hence if the acceleration time scale is shorter than the source crossing time, we expect $t_{\text {crs }}$ to smooth out $t_{\text {acc }}$. The dash-dotted line in Fig. 3 (right) shows the ratio of the time scales of $t_{\text {crs }} / t_{\text {cool }}$. we can see that $t_{\text {crs }}$ is longer than $t_{\text {acc }}$ below $\sim 2 \mathrm{keV}$, but comparable or shorter above $\sim 2 \mathrm{keV}$. As a result, for $1 \mathrm{ES} 1218+304$ it seems reasonable that the rise time of the flare is primary 
determined by the acceleration time of the electrons, while the fall time of the flare is due to the synchrotron cooling time scale. Only the rise time of the flare in the lower energy bands are dominated by the light crossing time $t_{\text {crs }}$, but further studies using more data are necessary to confirm this model.

We are grateful to Dr. L. Costamante for kindly providing us with the numerical values of the EBL effects on TeV gamma-ray spectra, and Dr. M. Sikora for many fruitful discussions. This work was supported, in part, by a Department of Energy contract to SLAC no. DE-AC3-76SF00515.
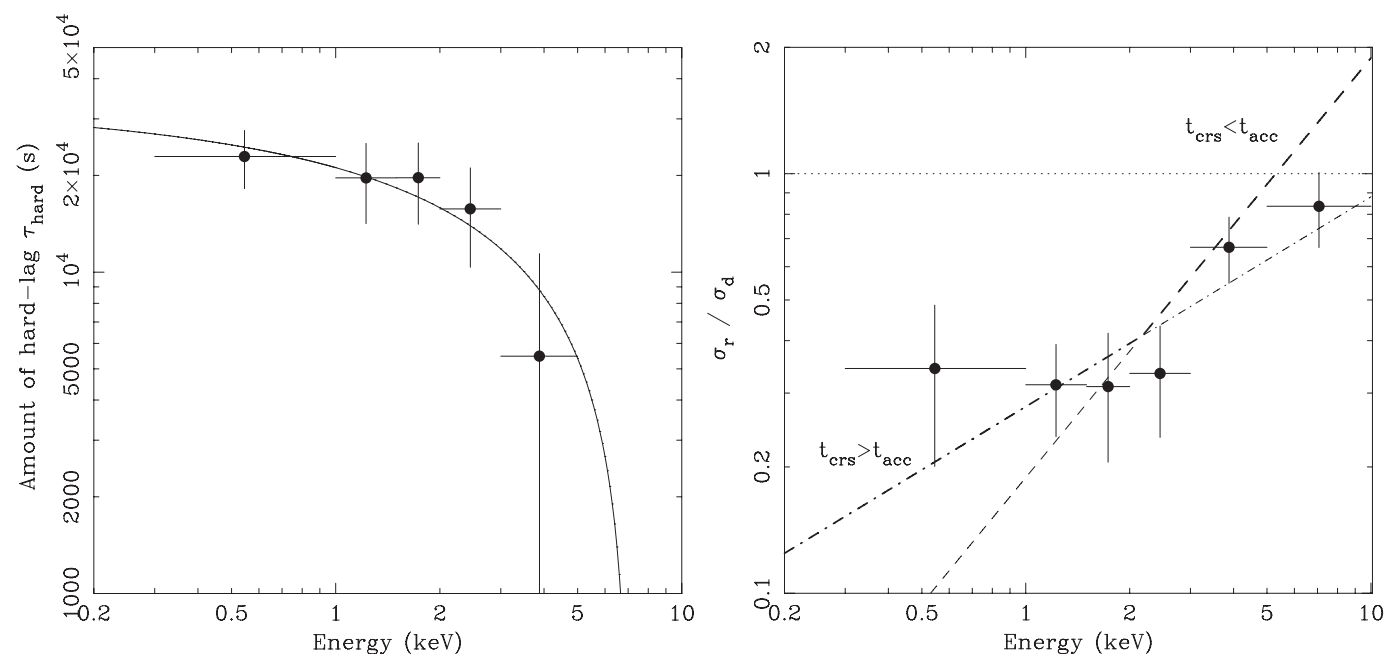

Fig. 3.- Left: Time lag of photons of various X-ray energy bands vs $5-10 \mathrm{keV}$ band photons. The solid line corresponds to a fit with $\tau_{\text {hard }}=9.65 \times 10^{-2}(1+z)^{3 / 2} \xi B^{-3 / 2} \delta^{-3 / 2}\left(7.1^{1 / 2}-E_{\text {low }}^{1 / 2}\right)$ where $\delta$ is fixed to 20.0. Right: Energy dependence of the pulse shape, defined as the ratio of $\sigma_{\mathrm{r}}$ and $\sigma_{\mathrm{d}}$. The dashed line shows the predicted value of $\sigma_{\mathrm{r}} / \sigma_{\mathrm{d}}$, calculated from $t_{\text {acc }} / t_{\text {cool }}=E / E_{\max } \sim E / 5.3 \mathrm{keV}$. The dash-dotted line shows the ratio of $t_{\text {crs }} / t_{\text {cool }}$.

\section{REFERENCES}

Albert, J., et al. 2006, ApJ, 642, L119

Albert, J., et al. 2007, ApJ, 669, 862

Aharonian, F. et al., 2007, ApJ, 664, L71

Brinkmann, W., Papadakis, I. E., Raeth, C., Mimica, P., \& Haberl, F., 2005, A\&A, 443, 397 
Chiaberge, M., \& Ghisellini, G. 1999, MNRAS, 306, 551

Costamante, L., et al. 2001, A\&A, 371, 512

Costamante, L., 2007, Ap\&SS, 309, 487 (astro-ph/0612709v1)

Edelson, R. A., \& Krolik, J. H. 1989, proceedings of the International Astronomical Union Symposium, 134, 96

Edelson, R., Griffiths, G., Markowitz, A., Sembay, S., Turner, M. J. L., \& Warwick, R. 2001, ApJ, 554, 274

Fortin, P. 2007, astro-ph/07093657

Fukazawa, Y., et al. 2006, Proc.SPIE, 6266, 75

Gruber, D. E., Matteson, J. L., Peterson, L. E., \& Jung, G. V. 1999, ApJ, 520, 124

Hufnagel, B. R., \& Bregman, J. N. 1992, ApJ, 386, 473

Inoue, S., \& Takahara, F. 1996, ApJ, 463, 555

Kataoka, J., et al. 1999, ApJ, 514, 138

Kataoka, J. 2000, Ph.D thesis, Univ. Tokyo (http://www.hp.phys.titech.ac.jp/kataoka/paperJK00-thesis.pc

Kataoka, J., et al. 2000, ApJ, 528, 243

Kataoka, J., et al. 2001, ApJ, 560, 659

Kirk, J. G., Rieger, F. M, \& Mastichiadis, A. 1998, A\&A, 333, 452

Kokubun, M. et al. 2007, PASJ, 59, S53

Koyama, K. et al. 2007, PASJ, 59, S23

Mitsuda, K. et al. 2007, PASJ, 59, S1

Norris, J. P., et al. 1996, ApJ, 459, 393

Primack, J. R., Somerville, E. S., Bullock, J. S. \& Devriendt, J. E. G. 2001, AIP Conf. Proc., 558,463

Primack, J. R., Bullock, J. S., \& Somerville, E. S. 2005, AIP Conf. Proc., 745, 23

Serlemitsos, P. J., et al. 2007, PASJ, 59, S9 
Stefan, R., et al. 2008, in prep

Takahashi, T. et al. 1996, ApJ, 470, L89

Takahashi, T. et al. 2000, ApJ, 542, L105

Takahashi, T. et al. 2007, PASJ, 59, S35

Tanihata, C. et al. 2001, ApJ, 563, 569

Ulrich, M-H., Maraschi, L., \& Urry, C. M. 1997, ARA\&A, 35, 445

Urry, C. M., \& Padovani, P. 1995, PASP, 107, 803

Veron-Cetty, M. P. \& Veron, P. 2003, A\&A, 412, 339

Zhang, Y. H., Cagnoni, I., Treves, A., Celotti, A., \& Maraschi, L. 2004, ApJ, 605, 98 